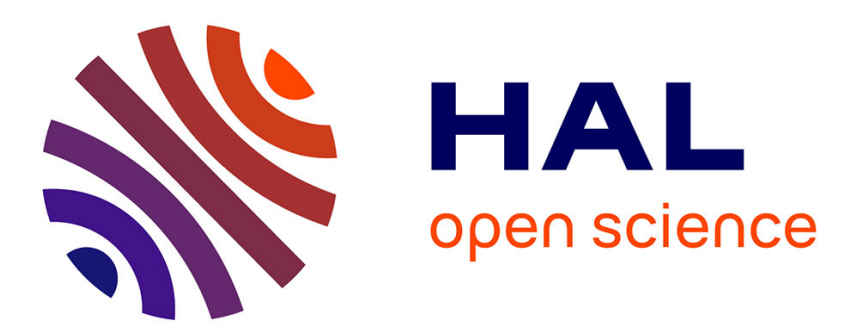

\title{
Some results on the global dynamics of the regularized restricted three-body problem with dissipation
}

\author{
Alessandra Celletti, Letizia Stefanelli, Elena Lega, Claude Froeschlé
}

\section{To cite this version:}

Alessandra Celletti, Letizia Stefanelli, Elena Lega, Claude Froeschlé. Some results on the global dynamics of the regularized restricted three-body problem with dissipation. Celestial Mechanics and Dynamical Astronomy, 2011, 109 (3), pp.265-284. 10.1007/s10569-010-9326-y . hal-00606644

\section{HAL Id: hal-00606644 https://hal.science/hal-00606644}

Submitted on 7 Jul 2011

HAL is a multi-disciplinary open access archive for the deposit and dissemination of scientific research documents, whether they are published or not. The documents may come from teaching and research institutions in France or abroad, or from public or private research centers.
L'archive ouverte pluridisciplinaire HAL, est destinée au dépôt et à la diffusion de documents scientifiques de niveau recherche, publiés ou non, émanant des établissements d'enseignement et de recherche français ou étrangers, des laboratoires publics ou privés. 


\title{
Some results on the global dynamics of the regularized restricted three-body problem with dissipation
}

\author{
Alessandra Celletti \\ Dipartimento di Matematica \\ Università di Roma Tor Vergata \\ Via della Ricerca Scientifica 1 \\ I-00133 Roma (Italy) \\ (celletti@mat.uniroma2.it) \\ Elena Lega
}

UNSA, CNRS UMR 6202, Observatoire de Nice,

Bv. de l'Observatoire, B.P. 4229

06304 Nice Cedex 4 (France)

(elena.lega@oca.eu)

\author{
Letizia Stefanelli \\ Dipartimento di Matematica \\ Università di Roma Tor Vergata \\ Via della Ricerca Scientifica 1 \\ I-00133 Roma (Italy) \\ (stefanel@mat.uniroma2.it)
}

\section{Claude Froeschlé}

UNSA, CNRS UMR 6202, Observatoire de Nice,

Bv. de l'Observatoire, B.P. 4229

06304 Nice Cedex 4 (France)

(claude.froeschledobs-nice.fr)

December 6, 2010

\begin{abstract}
We perform an analysis of the dynamics of the circular, restricted, planar three-body problem under the effect of different kinds of dissipation (linear, Stokes and PoyntingRobertson drags). Since the problem is singular, we implement a regularization technique in the style of Levi-Civita. The effect of the dissipation is often to decrease the semi-major axis; as a consequence the minor body collides with one of the primaries. In general, it is quite difficult to find non-collision orbits using random initial conditions. However, by means of the computation of the Fast Lyapunov Indicators (FLI), we obtain a global view of the dynamics. Precisely, we detect the regions of the phase space potentially belonging to basins of attraction. This investigation provides information on the different regions of the phase space, showing both collision and non-collision trajectories. Moreover, we find periodic orbit attractors for the case of linear and Stokes drags, while in the case of the Poynting-Robertson effect no other attractors are found beside the primaries, unless a fourth body is added to counterbalance the dissipative effect.
\end{abstract}

Keywords. Three-body problem, dissipative effects, regularization theory. 


\section{Introduction}

Dissipative effects play a key role in Solar System dynamics. One of the most important mechanisms of dissipation is the Poynting-Robertson (hereafter PR) effect (see [4]), which is due to the solar radiation incident on a particle. Numerical experiments on the dynamical consequences of the PR effect in a three-body problem have been carried out since the '80s (see, e.g., [8]). Another important kind of non gravitational effect is the so-called Stokes drag; this effect is due to the collisions of particles with the molecules of the gas nebula being present during the formation of a planetary system.

The main effect of the dissipation is that of reducing the semi-major axis of the particle, which is compelled to spiral toward the Sun. Resonances appear as the only mechanism which is able to slow down the particle's fall to the Sun; for this reason their role has been extensively studied in the framework of the three-body problem with PR or Stokes drags (see also [9] for applications to exoplanets). It has been shown that interior resonances (namely, taking place inside the orbit of the primary different from the Sun) cannot compensate the decrease of the semi-major axis of the particle (compare with [8]). Moreover, the stationary points $L_{4}$ and $L_{5}$ are shown to become unstable for both kinds of dissipation. An analytical study of the linearized stability of $L_{4}$ and $L_{5}$ is provided in [12], [13], while a numerical analysis of the 1:1 resonance, taking also into account the effect of the inclination and of the eccentricity, can be found in [11]. It was shown in [1], [2], [3], [15] that, in the case of PR and Stokes drags, exterior resonances (namely, outside the orbit of the other primary) may compensate the decrease of the semi-major axis and that stationary solutions still exist. Precisely, for the PR effect the capture into resonance turns out to be only temporary ([2], [15]), although on times long enough for statistical effects to be significant; for Stokes drag ([1]) stationary solutions appear to be stable. Finally, the influence on dust grains of exterior resonances with the Earth is investigated in [16], showing that some particles may be trapped, even for times of the order of $10^{5}$ years, in the planar, circular, restricted three-body problem.

The aim of the present paper is to study numerically the dynamics of the planar, circular, restricted three-body problem under the effect of the dissipation. We consider the cases of a linear drag, Stokes drag and PR effect (as a matter of fact, the linear drag is a special case of the Stokes drag). As a particular case, we have considered a particle moving under the gravitational effect of Sun and Jupiter. We investigate the existence of periodic attractors under the different dissipations; linear and Stokes drag admit periodic orbits of different periods, while we have not been able to determine periodic motions under the PR dissipation, unless a fourth body (e.g., a Saturn-like body) is present in the system. To have a global view of the dynamics, we compute the Fast Lyapunov Indicators (hereafter FLI, see [7], [6]), which require the integration of the equations of motion and of the variational equations. Since the equations of motion of the three-body problem are singular, particular care must be taken when performing the numerical integration, especially in the dissipative context where particles often move toward one of the singularities. To get rid of this problem we regularize the equations of motion by implementing the Levi-Civita transformation ([18], see also [5] and [17] for applications). 
The study of the regularized tangent space in the conservative case can be found in [10], while, as far as we know, this is the first analysis adopting regularization in the dissipative setting. The global analysis of the phase space provided by the FLI shows the effects of the different kinds of dissipation on the dynamics; we find that, even on short integration times, a large fraction of test particles with initial conditions in the interior region collides with the Sun. Due to the effect of the dissipation, orbits with close encounters with the Sun experience fast deflections on collision orbits. In the exterior region, collisions occur mainly with Jupiter. When increasing the strength of the dissipation or the integration time, a larger fraction of orbits collides with one of the primaries. In general, the PR effect is faster than that provoked by Stokes and linear drags. Through the computation of the FLI on quite long integration times in the Stokes case, we show that certain portions of the space phase remain stable, at least up to this time. Orbits in these regions are computed on much longer times providing the existence of attractors (different from the primaries) for some values of the parameter $\alpha$ (the ratio between the velocity of the gas and the keplerian velocity at a given radius) up to $\alpha=0.6$. For the physical value, for example $\alpha=0.995$ ([1]) used in the model of formation of the Solar System, we could find only temporary stability. An interesting result of our investigation is that even when dissipative effects are taken into account, there exist some regions where the particle stays save from collisions with one of the primaries.

This paper is organized as follows: in Section 2 we describe the model problem in the dissipative framework and the Levi-Civita regularization. Results on periodic attractors and on the global analysis are provided in Section 3. Conclusions are detailed in Section 4; the computation of the regularized dissipative equations is provided in the Appendix.

\section{The restricted three-body problem with dissipation}

We consider the planar, circular, restricted three-body problem, which is composed by three bodies, say an asteroid moving in the gravitational field of two primaries $P_{1}, P_{2}$, with masses, respectively, $1-\mu, \mu$. It is assumed that the motion of the three bodies takes place on the same plane, that the relative motion of the primaries is circular and that (restricted problem) the mass of the asteroid is so small that its influence on the primaries can be neglected. In the following sections we will describe the equations of motion in the conservative setting as well as with the effect of dissipation, both in the cartesian and regularized variables. In particular, we shall consider a linear drag, the Poynting-Robertson dissipation and the Stokes drag.

\subsection{Equations of motion in the conservative setting}

We briefly present the equations of motion in the conservative framework, together with the formulae necessary to derive the equations in the dissipative case as well as the corresponding regularized equations. Let us consider an inertial reference frame, say $(\mathcal{O}, \xi, \eta)$, with origin in the barycenter of the primaries, which are assumed to move on a plane. Let $(\xi, \eta)$ be the coordinates of the asteroid; denoting by $\left(\xi_{1}, \eta_{1}\right),\left(\xi_{2}, \eta_{2}\right)$ the coordinates of the primaries, the 
equations of motion of the asteroid can be written as ([12])

$$
\begin{aligned}
& \ddot{\xi}=\mu \frac{\xi_{1}-\xi}{r_{1}^{3}}+(1-\mu) \frac{\xi_{2}-\xi}{r_{2}^{3}} \\
& \ddot{\eta}=\mu \frac{\eta_{1}-\eta}{r_{1}^{3}}+(1-\mu) \frac{\eta_{2}-\eta}{r_{2}^{3}}
\end{aligned}
$$

where $r_{1}, r_{2}$ denote the distances from the primaries:

$$
r_{1}=\sqrt{\left(\xi_{1}-\xi\right)^{2}+\left(\eta_{1}-\eta\right)^{2}}, \quad r_{2}=\sqrt{\left(\xi_{2}-\xi\right)^{2}+\left(\eta_{2}-\eta\right)^{2}} .
$$

Let us introduce a synodic reference frame $(\mathcal{O}, x, y)$, rotating with the angular velocity $n$ of the primaries, where $n$ can be normalized to one, thanks to a proper choice of the units. Let us choose the axes of the synodic frame so that the coordinates of the primaries become $\left(x_{1}, y_{1}\right)=(-\mu, 0),\left(x_{2}, y_{2}\right)=(1-\mu, 0)$. The link between the synodic and the sidereal reference frames is

$$
\begin{aligned}
\xi & =x \cos (t)-y \sin (t) \\
\eta & =x \sin (t)+y \cos (t)
\end{aligned}
$$

while the distances of the asteroid from the primaries are now given by

$$
r_{1}=\sqrt{(x+\mu)^{2}+y^{2}}, \quad r_{2}=\sqrt{(x-1+\mu)^{2}+y^{2}} .
$$

Computing the second derivative of (2) with respect to time and inserting the result in (1) one obtains the equations of motion in the synodic frame:

$$
\begin{aligned}
\ddot{x} & =2 \dot{y}+x-(1-\mu) \frac{x+\mu}{r_{1}{ }^{3}}-\mu \frac{x-1+\mu}{r_{2}{ }^{3}} \\
\ddot{y} & =-2 \dot{x}+y-(1-\mu) \frac{y}{r_{1}{ }^{3}}-\mu \frac{y}{r_{2}{ }^{3}} .
\end{aligned}
$$

\subsection{Equations of motion in the dissipative case}

The Stokes drag is a force experienced by a particle moving in a gas, and it is due to the collisions of the particle with the molecules of the gas. In the frame of the protoplanetary nebula, Stokes drag is used to describe the dissipative force acting on a particle moving around a star. The gas is assumed to be in circular motion around the system's barycenter; its angular velocity is slightly smaller by a factor $\alpha$, than the Keplerian velocity at the same distance. The particle is assumed to be small or to move very slowly. In such a context, this effect can be described as a dissipative force acting on the particle, modeled by a linear function of the relative velocity of the particle with respect to the gas. In synodic coordinates the components $\left(F_{x}, F_{y}\right)$ of the dissipative force take the form ([12]):

$$
\left(F_{x}, F_{y}\right)=-k(\dot{x}-y+\alpha \Omega y, \dot{y}+x-\alpha \Omega x)
$$


where $k \in[0,1)$ is the dissipative constant, depending on several physical parameters ([1]) like the viscosity of the gas, the radius and the mass of the particle; $\Omega=\Omega(r) \equiv r^{-3 / 2}$ is the Keplerian angular velocity at distance $r=\sqrt{x^{2}+y^{2}}$ from the origin of the synodic frame and $\alpha \in[0,1)$ is the ratio between the gas and Keplerian velocities ([13]). The equations of motion with a Stokes dissipation read as

$$
\begin{aligned}
& \ddot{x}=2 \dot{y}+x-(1-\mu) \frac{x+\mu}{r_{1}{ }^{3}}-\mu \frac{x-1+\mu}{r_{2}{ }^{3}}-k(\dot{x}-y+\alpha \Omega y) \\
& \ddot{y}=-2 \dot{x}+y-(1-\mu) \frac{y}{r_{1}{ }^{3}}-\mu \frac{y}{r_{2}{ }^{3}}-k(\dot{y}+x-\alpha \Omega x) .
\end{aligned}
$$

Let us remark that the particular case $\alpha=0$ corresponds to the linear drag with a dissipative force given by

$$
\left(F_{x}, F_{y}\right)=-k(\dot{x}-y, \dot{y}+x) .
$$

The Poynting-Robertson force (see also [14]) is a non gravitational effect acting on a particle moving around the Sun and it is due to the solar radiation incident on the particle. In the synodic frame it takes the following form ([12]):

$$
\left(F_{x}, F_{y}\right)=-\frac{k}{r_{1}^{2}}\left(\dot{x}-y+\frac{x}{r_{1}^{2}}(x \dot{x}+y \dot{y}), \dot{y}+x+\frac{y}{r_{1}^{2}}(x \dot{x}+y \dot{y})\right) .
$$

One can split this force into two components. The first one is the drag component due to the impact of the photons of the solar radiation with the particle; it can be described as a force proportional and opposed to the velocity of the particle in the synodic frame, but with a magnitude proportional to the inverse of the square of the distance from the Sun (because the amount of light hitting the particle is proportional to the inverse of the square of the distance from the Sun):

$$
-\frac{k}{r_{1}^{2}}(\dot{x}-y, \dot{y}+x) \text {. }
$$

A second component represents the Doppler shift of solar radiation that hits the particle, due to the fact that the particle is moving around the Sun:

$$
-\frac{k}{r_{1}{ }^{4}}((x \dot{x}+y \dot{y}) x,(x \dot{x}+y \dot{y}) y) ;
$$

we do not consider this contribution, since it decreases much faster than the drag component, being proportional to the inverse of the fourth power of the distance from the Sun.

In conclusion, we consider the following equations of motion including the Poynting-Robertson drag:

$$
\begin{aligned}
\ddot{x} & =2 \dot{y}+x-(1-\mu) \frac{x+\mu}{r_{1}{ }^{3}}-\mu \frac{x-1+\mu}{r_{2}{ }^{3}}-\frac{k}{r_{1}{ }^{2}}(\dot{x}-y) \\
\ddot{y} & =-2 \dot{x}+y-(1-\mu) \frac{y}{r_{1}{ }^{3}}-\mu \frac{y}{r_{2}{ }^{3}}-\frac{k}{r_{1}^{2}}(\dot{y}+x)
\end{aligned}
$$

where $k \in[0,1)$ is the dissipative constant. 


\subsection{Regularized equations of motion}

Since the equations of motion are singular at the primaries' locations, we now address the question of writing the equations of motion in regularized variables ([18], [5]). Using standard regularization theory, we define a change of coordinates, known as the Levi-Civita transformation, by introducing the parametric coordinates $\left(u_{1}, u_{2}\right)$ through the expression

$$
\begin{aligned}
x & =u_{1}^{2}-u_{2}{ }^{2}+x_{0} \\
y & =2 u_{1} u_{2},
\end{aligned}
$$

where $x_{0}$ is the coordinate of the singularity, namely $x_{0}=-\mu$ to regularize around $P_{1}$ and $x_{0}=1-\mu$ to regularize around $P_{2}$. Next, we define a change of time by introducing the regularized time $s$ related to the ordinary time $t$ through the expression

$$
d t=R d s, \quad R \equiv u_{1}^{2}+u_{2}^{2} .
$$

The regularized equations of motion read as

$$
\begin{aligned}
& u_{1}^{\prime \prime}=\left[\frac{1}{R}\left(u_{1}^{\prime 2}+u_{2}^{\prime 2}-\frac{1-\mu_{s}}{2}\right)+\frac{R}{2}\left(R+x_{0}-\mu_{s} \frac{R-x_{s}}{r_{s}^{3}}\right)\right] u_{1}+2 R u_{2}^{\prime}+F_{u_{1}} \\
& u_{2}^{\prime \prime}=\left[\frac{1}{R}\left(u_{1}^{\prime 2}+u_{2}^{\prime 2}-\frac{1-\mu_{s}}{2}\right)+\frac{R}{2}\left(R-x_{0}-\mu_{s} \frac{R+x_{s}}{r_{s}^{3}}\right)\right] u_{2}-2 R u_{1}^{\prime}+F_{u_{2}},
\end{aligned}
$$

where $r_{s}=r_{2}, \mu_{s}=\mu, x_{s}=1, x_{0}=-\mu$ if we regularize in $P_{1}$ and $r_{s}=r_{1}, \mu_{s}=$ $1-\mu, x_{s}=-1, x_{0}=1-\mu$ if we regularize in $P_{2}$. The dissipative terms $\left(F_{u_{1}}, F_{u_{2}}\right)$ are zero in the conservative case, while in the dissipative case they take the following expression:

$$
\begin{array}{rlr}
\left(F_{u_{1}}, F_{u_{2}}\right) & =\left(-k \frac{R}{2}\left[2 u_{1}^{\prime}-\left(R-x_{0}\right) u_{2}\right],-k \frac{R}{2}\left[2 u_{2}^{\prime}-\left(R+x_{0}\right) u_{1}\right]\right) \quad \text { (linear drag) } \\
\left(F_{u_{1}}, F_{u_{2}}\right) & =\left(-k \frac{R}{2}\left[2 u_{1}^{\prime}-\left(R-x_{0}\right) u_{2}+\alpha \Omega\left(R-x_{0}\right) u_{2}\right],\right. & \\
& \left.-k \frac{R}{2}\left[2 u_{2}^{\prime}-\left(R+x_{0}\right) u_{1}-\alpha \Omega\left(R+x_{0}\right) u_{1}\right]\right) & \text { (Stokes drag). }
\end{array}
$$

Concerning the PR effect, since it is singular in $r_{1}$ we need to distinguish two different cases:

- if we regularize in $P_{1}$ then:

$$
\left(F_{u_{1}}, F_{u_{2}}\right)=\left(-\frac{k}{2 R}\left(2 u_{1}^{\prime}-u_{2}\left(R-x_{0}\right)\right),-\frac{k}{2 R}\left(2 u_{2}^{\prime}+u_{1}\left(R+x_{0}\right)\right)\right) ;
$$

- if we regularize in $P_{2}$ then:

$$
\left(F_{u_{1}}, F_{u_{2}}\right)=\left(-k \frac{R}{2 r_{1}^{2}}\left(2 u_{1}^{\prime}-u_{2}\left(R-x_{0}\right)\right),-k \frac{R}{2 r_{1}^{2}}\left(2 u_{2}^{\prime}+u_{1}\left(R+x_{0}\right)\right)\right) .
$$

A detailed derivation of the equations of motion in the regularized variables is provided in the Appendix. 


\section{Periodic orbits and global behavior}

\subsection{Periodic orbits in the dissipative setting}

We investigate the existence of periodic attractors under the different kinds of dissipation introduced in Section 2. We assume that the primaries' mass ratio amounts to $9.537 \cdot 10^{-4}$ (namely, the mass of Jupiter over the total mass of the primaries). The periodic orbits are determined by applying a Newton's method on the Poincaré map associated to the equations of motion (for example one can take the Poincaré section by considering the crossing points of the trajectories with the surface $y=0$, for instance); a continuation method is applied to find the periodic orbits for different values of the parameters. In practical computations, we start by selecting a grid of initial conditions in the orbital elements $(a, e)$, being $a$ the semimajor axis and $e$ the orbital eccentricity. Then we derive the initial conditions through the following algorithm. We first compute the energy $E$ and the angular momentum $L$ by means of $E=-1 /(2 a), L=\sqrt{\left(1-e^{2}\right) /(-2 E)}$. Then we select initial conditions of the form $\left(x(0), y(0), p_{x}(0), p_{y}(0)\right)=\left(x_{0}, 0,0, p_{y, 0}\right)$ with

$$
x_{0}=\frac{1 \pm \sqrt{1+2 E L^{2}}}{-2 E}, \quad p_{y, 0}=\frac{L}{x_{0}} .
$$

By a Newton's method we look for the fixed point on the Poincare map starting from the initial condition $\left(x_{0}, 0,0, p_{y, 0}\right)$. Next we compute the period of the solution and we mark the orbital elements $(a, e)$ of the initial grid, which lead to a trajectory with given period. This strategy allows us to determine the domain of convergence of Newton's method in the element's plane $(a, e)$.

We start by looking for periodic orbits in the conservative case $k=0$ and then we proceed to analyze the dissipative case. In order to compute the occurrence of the periodic orbits and to draw the domain of convergence of Newton's method, we select a grid of $100 \times 100$ initial conditions in the orbital elements with $0<a \leq 6$ (remind that the distances between the primaries has been set to one) and $0<e \leq 0.5$. For each initial condition, we implement a Newton's method to look for fixed points on the Poincaré section obtained by setting $y=0$. The results are shown in Figure 1, which provides the domain of convergence of Newton's method in the conservative case with $k=0$ : the left picture shows a grid over the semimajor axis up to 6 times the Jupiter-Sun distance and for eccentricities up to 0.5; the right panel shows a grid for the semimajor axis between 0 and 1 . Almost all solutions are represented by the periodic orbits with periods $3,4,5$, but there occur also a few periodic orbits with different periods. For $k$ different from zero a continuation method can be implemented to detect periodic attractors, which are found within the linear and Stokes drag models, while they do not occur when using the three-body problem with the Poynting-Robertson drag. Figure 2 shows two periodic orbits of period 3 and 4 in the conservative case and in the linear drag case with $k=10^{-5}$; the corresponding initial conditions are provided in Table 1 .

Stokes dissipation depends on the parameter $\alpha$, which represents the ratio between the gas and Keplerian velocities. Using a small value, for example $\alpha=0.05$, one can find the same periodic orbits of the linear case. Increasing $\alpha$ these orbits are modified (Figure 3, left) and 

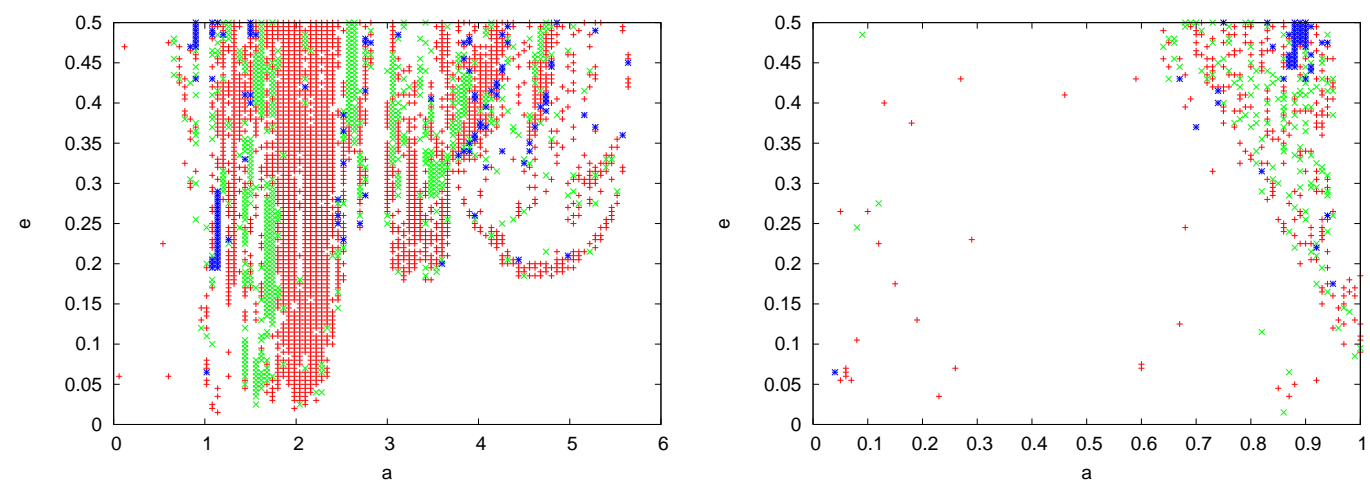

Figure 1: Domain of convergence of Newton's method in the conservative case: + denotes the periodic orbit of period $3, \times$ refers to period 4 and $*$ to period 5 (respectively, red, green, blue on the on-line version). Left: a grid over the semimajor axis between 0 and 6. Right: a grid over the semimajor axis between 0 and 1 .

\begin{tabular}{|lllll|}
\hline Dissipation & $x(0)$ & $\dot{x}(0)$ & $y(0)$ & $\dot{y}(0)$ \\
\hline \hline Figure 2, left & 0.63494347 & -0.99563772 & -1.19873351 & 0.18354021 \\
Figure 2, right & -1.75550227 & 0.19093344 & $-4.67132085 \cdot 10^{-15}$ & 1.26477867 \\
Figure 3, $\alpha=0.05$ & -2.07095471 & -0.272901991 & $-8.57062131 \cdot 10^{-16}$ & 1.713523845 \\
Figure 3, $\alpha=0.1$ & -2.10810068 & -0.270733105 & $-9.39068924 \cdot 10^{-15}$ & 1.773488031 \\
Figure 3, $\alpha=0.11$ & -2.11821224 & -0.270126714 & $-8.26276782 \cdot 10^{-15}$ & 1.789917779 \\
\hline
\end{tabular}

Table 1: Initial conditions of Figures 2 and 3, left.

they ultimately disappear when the parameter is too big, as it happens for $\alpha=0.12$. Let us remark that $\alpha=0.12$ is much lower than the typical physical value used for the model of formation of the solar system (e.g., $\alpha=0.995$ as in [1]). A question remains about the dynamical stability of this kind of orbits; this task is approached through the global analysis of the dynamics performed in Section 3.3.

\subsection{Periodic orbits in a 4-body problem}

So far we have seen that no periodic orbits can be found when adding the Poynting-Robertson drag; in this case, in order to find periodic orbits, one needs to modify the model, for example adding a third primary (e.g., a Saturn-like body), whose influence balances that of the two main primaries. More specifically, we introduce a four-body model composed by the primaries $P_{1}$, $P_{2}, P_{3}$ with masses $\mu_{1}, \mu_{2}, \mu_{3}$ respectively ( $P_{1}$ is the inner body and $P_{3}$ the outermost one). Let $S$ be a minor body moving in the gravitational field of the primaries. We consider an 

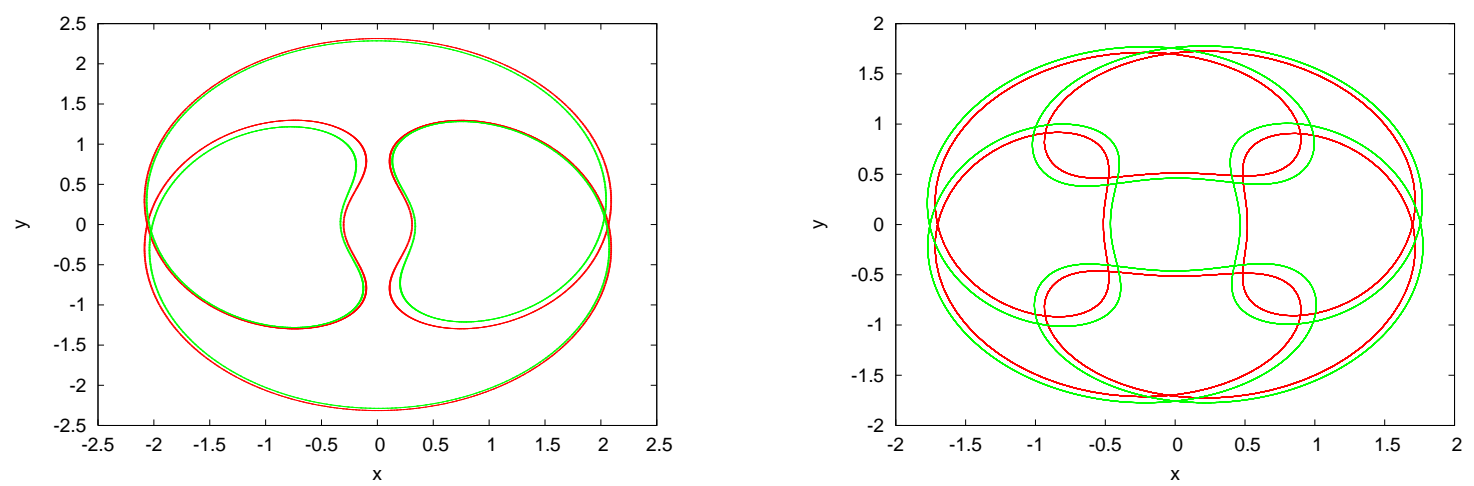

Figure 2: Periodic orbits of period 3 (left) and 4 (right) for the conservative case (inner curve) and under the linear drag with $k=10^{-5}$ (outer curve).

inertial reference frame with origin $\mathcal{O}$ coinciding with the barycenter of $P_{1}, P_{2}$ and $P_{3}$. Let $r_{j}$ be the distances of $P_{j}$ from $\mathcal{O}$ for $j=1,2,3$. As a first approximation we assume that the three primaries move on fixed circular orbits around the origin, so that $r_{2}-r_{1}=1$ and that $r_{3}-r_{1}=\beta$ for some constant $\beta>0$; the crude assumption that we do not consider the interaction between Jupiter and the Saturn-like body represents a first approximation of the real problem, which indeed should take into account also the mutual interaction between the two planets. Let us normalize the units such that the frequency of $P_{2}$ is unity, which implies that $\mu_{1}+\mu_{2}=1$ and that the distance between $P_{1}$ and $P_{2}$ is unity. By definition of the origin we also have that $\mu_{1} r_{1}+\mu_{2} r_{2}+\mu_{3} r_{3}=0$. Let $\left(X_{j}, Y_{j}\right)$ be the coordinates (depending on time) of $P_{j}, j=1,2,3$, and let $(x, y)$ be the coordinates of the asteroid, whose dynamics is described by the Hamiltonian function

$$
H\left(p_{x}, p_{y}, x, y, t\right)=\frac{1}{2}\left(p_{x}^{2}+p_{y}^{2}\right)-R(x, y, t),
$$

where $p_{x}, p_{y}$ are the momenta conjugated to the coordinates and

$R(x, y, t) \equiv \frac{\mu_{1}}{\sqrt{\left(x-X_{1}\right)^{2}+\left(y-Y_{1}\right)^{2}}}+\frac{\mu_{2}}{\sqrt{\left(x-X_{2}\right)^{2}+\left(y-Y_{2}\right)^{2}}}+\frac{\mu_{3}}{\sqrt{\left(x-X_{3}\right)^{2}+\left(y-Y_{3}\right)^{2}}}$.

Let us identify $P_{1}$ with the Sun, $P_{2}$ with Jupiter and $P_{3}$ with Saturn; since Jupiter and Saturn move approximately in a $5: 2$ orbital resonance, by Kepler's laws we can set $\beta=\left[2 \pi \cdot \frac{5}{2}\right]^{2 / 3}$. Moreover, setting $\mu_{2}=\mu, \mu_{1}=1-\mu$ for some $\mu>0$, one obtains that

$$
r_{1}=-\frac{\mu+\mu_{3} \beta}{1+\mu_{3}}, \quad r_{2}=\frac{1-\mu+\mu_{3}(1-\beta)}{1+\mu_{3}}, \quad r_{3}=\frac{\beta-\mu}{1+\mu_{3}} .
$$

Let us now introduce a synodic reference frame, rotating with the angular velocity of $P_{2}$. To this end, we introduce the generating function

$$
W\left(p_{x}, p_{y}, q_{1}, q_{2}, t\right) \equiv p_{x} q_{1} \cos t-p_{x} q_{2} \sin t+p_{y} q_{1} \sin t+p_{y} q_{2} \cos t,
$$



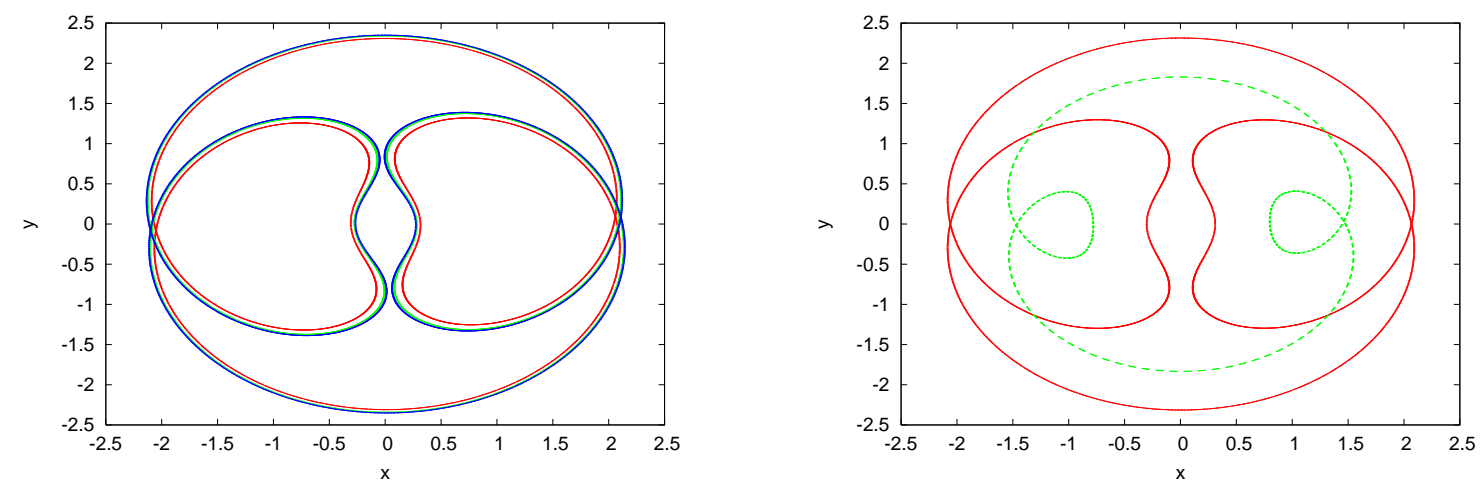

Figure 3: Left: Periodic orbits of period 3 with Stokes drag for $k=10^{-5}$ and $\alpha=0.05$ (inner curve), $\alpha=0.1$ (intermediate curve), $\alpha=0.11$ (external curve). Right: Periodic orbits of period 3 in the conservative case (external curve) and with Poynting-Robertson drag, using the Sun-Jupiter-Saturn model (inner curve) with $k=10^{-4}$ and $\mu_{3}=10^{-5} \mu$.

such that

$$
\begin{aligned}
x & =q_{1} \cos t-q_{2} \sin t \\
y & =q_{1} \sin t+q_{2} \cos t \\
p_{x} & =p_{1} \cos t-p_{2} \sin t \\
p_{y} & =p_{1} \sin t+p_{2} \cos t .
\end{aligned}
$$

Due to the assumptions on the circular motion of the primaries, we can set

$$
\begin{aligned}
X_{1} & =r_{1} \cos t & Y_{1} & =r_{1} \sin t \\
X_{2} & =r_{2} \cos t & Y_{2} & =r_{2} \sin t \\
X_{3} & =r_{3} \cos \left(\frac{2}{5} t\right) & Y_{3} & =r_{3} \sin \left(\frac{2}{5} t\right) .
\end{aligned}
$$

Therefore the new Hamiltonian is given by $H^{\prime}=H-\frac{\partial W}{\partial t}$, namely

$$
H^{\prime}\left(p_{1}, p_{2}, q_{1}, q_{2}, t\right)=\frac{1}{2}\left(p_{1}^{2}+p_{2}^{2}\right)+p_{1} q_{2}-p_{2} q_{1}-R^{\prime}\left(q_{1}, q_{2}, t\right)
$$

where

$$
\begin{aligned}
R^{\prime}\left(q_{1}, q_{2}, t\right) & \equiv \frac{\mu_{1}}{\sqrt{\left(q_{1}-r_{1}\right)^{2}+q_{2}^{2}}}+\frac{\mu_{2}}{\sqrt{\left(q_{1}-r_{2}\right)^{2}+q_{2}^{2}}} \\
& +\frac{\mu_{3}}{\sqrt{q_{1}^{2}+q_{2}^{2}+r_{3}^{2}-2 r_{3}\left(q_{1} \cos \left(\left(\frac{2}{5}-1\right) t\right)+q_{2} \sin \left(\left(\frac{2}{5}-1\right) t\right)\right.}}
\end{aligned}
$$

Next, we write the equations of motion associated to the above Hamiltonian and then we add the dissipative component. The periodic orbits are determined using a continuation method 
from the conservative case with $k=0$. As starting point, the orbital elements of Figure 1 have been considered.

The existence of periodic orbits is confirmed in the linear and Stokes cases; periodic attractors are also found when adding the Poynting-Robertson effect. Indeed, Figure 3 (right) shows the usual periodic orbit of period 3 of the conservative case and the periodic attractor (again with period 3), which is found using the Poynting-Robertson dissipation with $k=10^{-4}$ and setting $\mu_{3}=10^{-5} \mu$, being $\mu=9.537 \cdot 10^{-4}$ (notice that the mass of $P_{3}$ is smaller than the astronomical value for the mass of Saturn).

\subsection{Analysis of the dynamics}

A global behavior of the dynamics can be obtained through the analysis of the so-called Fast Lyapunov Indicator, hereafter FLI ([7], [6]), which is strictly related to the computation of the largest Lyapunov exponent. Its definition is based on the fact that the norm of the tangent vector over a finite interval of time is an indicator of the dynamical character of a trajectory (periodic, quasi-periodic or chaotic). We denote by $\underline{z}(0) \equiv(x(0), \dot{x}(0), y(0), \dot{y}(0))$ the vector of the initial conditions and let $\underline{v}(0) \in \mathbf{R}^{4}$ be an initial unit vector. Given a set of differential equations:

$$
\frac{d \underline{z}}{d t}=\underline{F}(\underline{z}), \quad \underline{z}=(x, \dot{x}, y, \dot{y}),
$$

the time evolution of the tangent vector $\underline{v}(t)$ is obtained by integrating the variational equations

$$
\frac{d \underline{v}}{d t}=\left(\frac{\partial \underline{F}}{\partial \underline{z}}\right) \underline{v}
$$

where $\frac{\partial \underline{F}}{\partial \underline{z}}$ is the Jacobian matrix. We define the quantity FLI up to a given time $T>0$ as

$$
\operatorname{FLI}(\underline{z}(0), \underline{v}(0), T) \equiv \log \|\underline{v}(T)\|
$$

where $\|\cdot\|$ denotes the Euclidean norm. We compute the FLI for different initial conditions $\underline{z}(0)$; its value is represented through a color scale, where the colors have the following meaning:

- chaotic motions, which admit very high FLI, are represented by the light grey color ${ }^{1}$,

- collision orbits with one of the primaries are marked in black,

- the remaining values of the FLI, with colors ranging from grey to dark grey correspond to orbits which are either stable, i.e. orbits which end up on an attractor, or quasi-stable, i.e. orbits needing a time longer than the integration time $T$ to be destabilized.

\footnotetext{
${ }^{1}$ In the electronic version of the paper the reader can find color pictures: light grey will appear as yellow, grey to dark grey colors will correspond from orange to blue colors.
} 
We remark that in order to compute the FLI we first integrate the regularized equations of motion and the regularized equations for the evolution of the tangent vector, which are obtained using the changes of coordinates described in the Appendix. When the time $T$ is reached, we use the inverse transformation to go back to the physical space. Finally we calculate the FLI as the logarithm of the norm of the tangent vector in the original coordinates.

The following set of figures is obtained by computing the FLI for a grid of $480 \times 640$ initial conditions regularly spaced in the $x, y$ coordinates with $-1.5<x(0)<1.5$ and $-1.125<$ $y(0)<2.875$. The other initial conditions are $\dot{y}=0$, while $\dot{x}$ is computed from the value of the Jacobi constant for the conservative case, which was set to 2.99047 , i.e. the value of the Jacobi constant at the Lagrangian points $L_{4}$ and $L_{5}$ with $\dot{x}=0$ and $\dot{y}=0$. We remark that for this value of the Jacobi constant there are no zero velocity curves, i.e no forbidden regions on the $x-y$ physical space. The integration time is set equal to $T=100$. The initial values of the tangent vectors are the same for all the orbits of the grid in the physical coordinates. Figure 4 provides the FLI for the conservative three-body problem. The left panel corresponds to the computation of the FLI using the non regularized equations, while on the right panel the computation is done with the regularized equations. In both cases we use a Runge-KuttaMerson integration scheme (namely, a fourth order Runge-Kutta method with adaptive time step for error control).
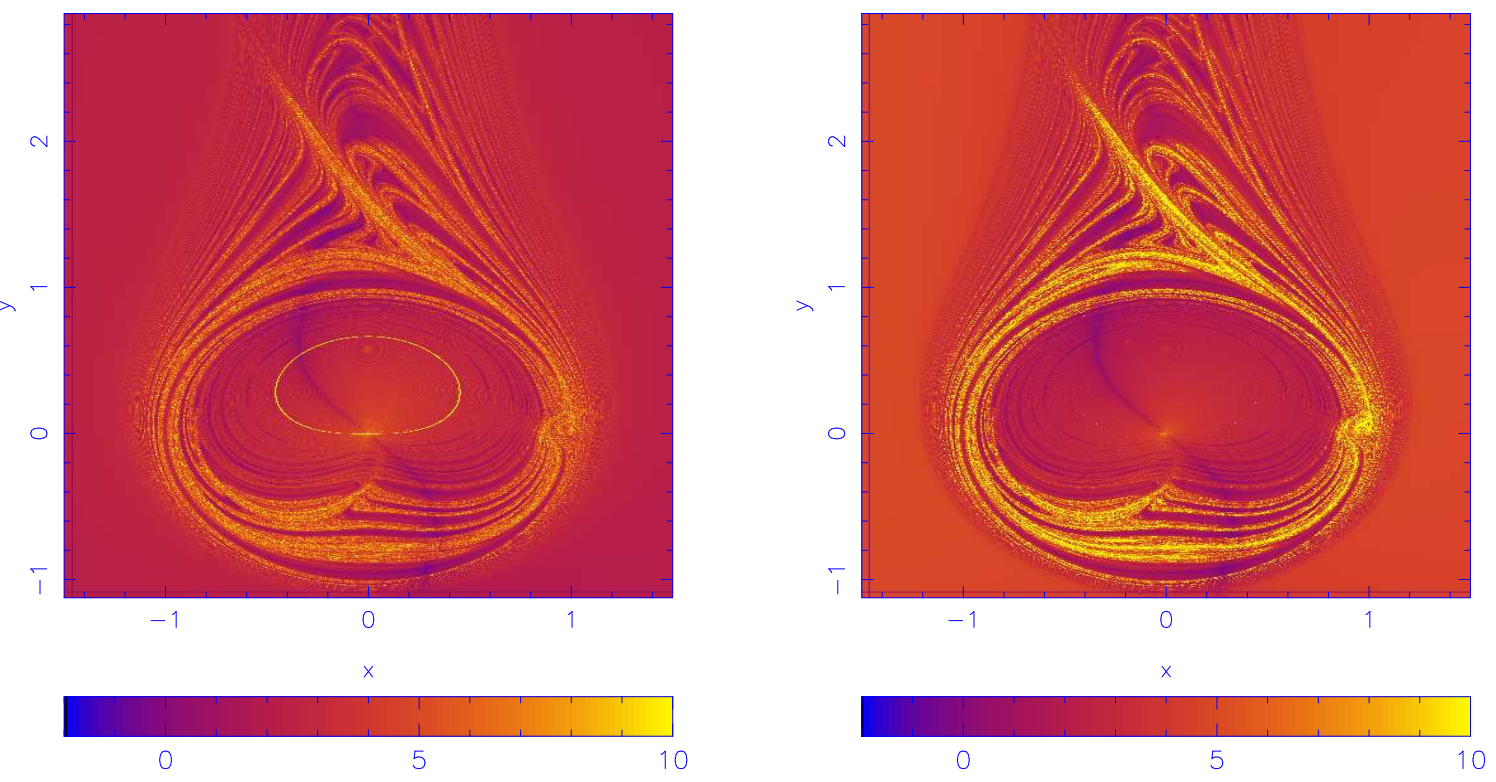

Figure 4: FLI map for the conservative case. The map is obtained integrating a grid of $480 \times 640$ orbits, with an integration time $T=100$. Each point in the map corresponds to an initial condition in the $x-y$ plane. We set $\dot{y}(0)=0$ and we calculate $\dot{x}(0)$ from the value of the Jacobi constant, set to 2.99047 which is the value at the Lagrangian point $L_{4}$ or $L_{5}$ with $\dot{x}=0, \dot{y}=0$. Left: non regularized equations; right: regularized equations. 
Both panels of Figure 4 reveal the presence of a large regular zone in the interior region with respect to the 1:1 resonance, while a peculiar light grey structure reveals a chaotic dynamics in the exterior region. When using non regularized equations, a light grey ring appears in the middle of the regular interior zone. The orbits with initial conditions on the light grey ring are those having the closest approach with the Sun within the time $T=100$. A check on the preservation of the Jacobi constant $C$ shows that for these orbits we obtain the worst energy preservation, i.e. the relative variation with respect to the initial value $C(0)$ is of the order of $10^{-5}$, while it is lower than $10^{-12}$ for all other orbits. This problem is due to the existence of the singularity and it cannot be canceled, neither by changing the integration scheme, nor through a better precision computation. Actually, the singularity means that the solution cannot be expressed as a power series development about it, while usual integration schemes are based on the development in power series of the solution. By using regularization, the problem is solved and the light grey ring disappears. In the following we study the dissipative dynamics using always the regularized equations.

Figure 5 refers to the three-body problem with Stokes drag for $\alpha=0.995$ (top) and PR-drag (bottom) with $T=100$, while Figure 6 refers to Stokes drag with a longer integration time, namely $T=5000$. Each picture is computed for two values of the dissipative constant, namely $k=10^{-5}$ (left panels) and $k=10^{-3}$ (right panels). In each panel we observe black zones, which represent collision orbits (namely orbits which come closer than $10^{-2}$ to one of the primaries). There is a wide black zone in the interior region: indeed, the orbits in this zone are those with the closest approach to the Sun in the conservative case. Therefore, this is the region of the phase space in which the dissipation, even a weak one $\left(k=10^{-5}\right)$, affects in a very short time $(T=100)$ the dynamics. Of course the size of the black region gets larger as we increase the dissipation (right panels) and the integration time (Figure 6). In the case of Poynting-Robertson drag the region of collision orbits turns out to be much larger than that appearing in the case of the Stokes drag. This is probably due to the dependence of the Poynting-Robertson drag on the inverse of the (square) distance from the Sun.

The light grey boundary of the collision region with the Sun in Figure 5 (bottom left) corresponds to orbits which are interpreted as chaotic, but whose distance remains larger than $10^{-2}$ from the Sun within $T=100$. In all pictures we also observe orbits colliding with Jupiter; they appear as black structures within the chaotic exterior region. When considering a longer integration time, say $T=5000$, we find that the chaotic exterior region is almost completely filled by orbits colliding with Jupiter for $k=10^{-5}$ (Figure 6, left panel); on the contrary, for $k=10^{-3}$ (Figure 6, right panel) the fraction of orbits colliding with Jupiter turns out to be smaller than that for $k=10^{-5}$. It is also interesting to observe that at $T=5000$ we still observe, in the exterior region, stable or quasi-stable zones (grey colors), which are almost unchanged with respect to the computation for $T=100$. Indeed, as shown in Figure 1, periodic orbits are typically found outside the orbit of Jupiter, say for $a \geq 1$. A detailed computation on longer times for some initial conditions with FLI values lower than 3 for $\alpha=0.995$ shows that these orbits become unstable on times larger than $T=5000$. However it is remarkable that on quite long times, we can detect regions of temporary stability through the FLI. 


\begin{tabular}{|lllll|}
\hline Orbit label & $x(0)$ & $\dot{x}(0)$ & $y(0)$ & $\dot{y}(0)$ \\
\hline \hline $\mathrm{A}$ & -2.09 & -0.6071558 & -0.5 & 1.713523845 \\
$\mathrm{~B}$ & -2.1 & -1.043882 & -1. & 1.713523845 \\
$\mathrm{C}$ & -1.9 & 0 & 0 & 1.713524 \\
\hline
\end{tabular}

Table 2: Initial conditions of Figure 8.

\subsection{Regions of stability and basins of attractions}

The aim of this section is to use the FLI on long integration times $(T=5000)$ in order to find orbits within given basins of attraction. To this end, we have considered a neighborhood of the periodic orbit of Figure 3 (left) for $\alpha=0.05$. Precisely, we have computed the FLI up to $T=5000$ for a set of $500 \times 500$ initial conditions regularly spaced in $x \in[-2.3,-1.8]$, $y \in[-1,0.5]$ with $\dot{y}=1.713523845$ (see Table 1, line 3) and $\dot{x}$ obtained from the Jacobi constant of the periodic orbit of Figure 3 (left) with $\alpha=0.05$ (namely $C=2.24409690$ ). In Figure 7 (left panel) we observe different regions (grey to dark grey colors, indicating stability up to $T=5000$ ) of various shapes embedded in a large collisional zone. A white circle denotes the initial conditions for $x$ and $y$ of the periodic orbit of Figure 3 (left). It is interesting to observe that the same FLI computation for $\alpha=0.995$ (Figure 7, right panel) provides the same zones of stability up to $T=5000$ as in the case with $\alpha=0.05$. A much longer integration time would be necessary to confirm the stability of such regions, but this would require months of CPU time. Indeed, the FLI values are useful to select initial conditions of orbits potentially belonging to basins of attraction. For example, by choosing FLI values lower than 3, which seems to be a good experimental threshold (compare with points A, B and C in Figure 7; see Table 2 for the corresponding initial conditions), we found orbits going on different periodic or point attractors for $\alpha=0.05$ (Figure 8, left column), while for $\alpha=0.995$ we observe only a transitory attracting behavior (Figure 8, right column). Precisely the initial condition labeled by A belongs to the basin of attraction of the periodic orbit of period three of Figure 3 (left), while the orbit B belongs to the basin of attraction of another periodic attractor and the orbit $\mathrm{C}$ goes to an attracting point. The computation of the Largest Characteristic Number (whose limit as time goes to infinity gives the Largest Lyapunov exponent) allows us to confirm on a time $T=10^{6}$ the stability of these orbits in the case $\alpha=0.05$ (Figure 9). Moreover, increasing the value of $\alpha$, we have found that the orbit A belongs to the basin of attraction of the periodic orbit of period three of Figure 3 (left) up to $\alpha=0.25$, the orbit B belongs to the same periodic attractor up to $\alpha=0.5$, while the orbit $\mathrm{C}$ goes to an attracting point up to $\alpha=0.6$. In conclusion, through the FLI charts we have a good indication of the potentially stable zones and of their shape. A detailed integration of single orbits allows us to confirm the long-term stability and the nature of the attractor to which they belong. 


\section{Conclusions}

Using the regularized equations for the computation of the equations of motion and of the variational equations, we performed a global study of the dynamics of the circular, restricted, three-body problem under the effect of different kinds of dissipation. As it is well known, the effect of the dissipation is that of decreasing the semi-major axis of orbits that collide with one of the primaries. We have recovered this result, showing which are the regions of the phase space affected by the dissipation even on very short time scales. Precisely, we found that a large fraction of test particles with initial conditions in the interior region collides with the Sun. Regular orbits having the closest approach with the Sun in the conservative setting are the first to be affected by the dissipation. In the exterior region, collisions occur mainly with Jupiter. When increasing the strength of the dissipation or the integration time, a larger fraction of orbits ends on one of the primaries. In general, the effect of the PR drag is faster than those associated to Stokes and linear drags. Moreover, we have found periodic orbit attractors for the case of the linear and Stokes drags, while in the case of the Poynting-Robertson effect no other non-trivial attractor (namely, not coinciding with one of the primaries) is found. In the latter case, in order to find periodic orbits, we need to modify the model, for example adding the action of a third primary (e.g., a Saturn-like body), whose effect turns out to balance that of the two main primaries. In the case of the Stokes drag, a long time ( $T=5000)$ FLI computation allows us to identify the size and the shape of the regions of the phase space potentially belonging to basins of attraction. A longer integration of individual orbits is necessary to confirm the attracting dynamics. For the rather unphysical case $\alpha=0.05$ we have found sizeable regions behaving as basins of attraction. For moderate values of $\alpha \leq 0.6$ we still find periodic attractors or points attractors, while for the more physical case $\alpha=0.995$ the stable behaviour turns out to be only temporary. It is remarkable to find that even in the presence of drag there are regions of the phase space, where the dynamics prevents the particle to collide with one of the primaries.

Acknowledgments. A.C. and L.S. acknowledge the grants ASI "Studi di Esplorazione del Sistema Solare" and PRIN 2007B3RBEY "Dynamical Systems and Applications" of MIUR. FLI computations have been done on the "Mesocentre SIGAMM" machine, hosted by the Observatoire de la Cote d'Azur.

\section{Appendix: The regularized equations}

The equations of motion of the three-body problem present two singularities at the primaries' positions, namely at $r_{1}=0$ and $r_{2}=0$. In order to study the motion at collision or at close encounters with the primaries one can implement regularization theory ([18], see also [5]). The classical technique consists of a change of coordinates, a rescaling of the time, and the use of energy preservation; the last ingredient must be avoided in the case of a dissipative system. We provide here the details of the regularization of the equations of motion including the dissipative terms. 
Let us write the equations of motion as

$$
\begin{aligned}
\ddot{x} & =2 \dot{y}+x-(1-\mu) \frac{x+\mu}{r_{1}{ }^{3}}-\mu \frac{x-1+\mu}{r_{2}{ }^{3}}+F_{x} \\
\ddot{y} & =-2 \dot{x}+y-(1-\mu) \frac{y}{r_{1}{ }^{3}}-\mu \frac{y}{r_{2}{ }^{3}}+F_{y},
\end{aligned}
$$

where $F_{x}, F_{y}$ denote the two components of the dissipative force, whose explicit expression is given in (3), (4), (5). We start by implementing the Levi-Civita transformation, which relates the cartesian coordinates $(x, y)$ to new variables $\left(u_{1}, u_{2}\right)$ according to the following formulae:

$$
\left(\begin{array}{l}
x \\
y
\end{array}\right)=\left(\begin{array}{rr}
u_{1} & -u_{2} \\
u_{2} & u_{1}
\end{array}\right)\left(\begin{array}{l}
u_{1} \\
u_{2}
\end{array}\right)+\left(\begin{array}{c}
x_{0} \\
0
\end{array}\right),
$$

where $x_{0}$ is the abscissa of the collision point that we will regularize, i.e. $x_{0}=-\mu$ for collisions with $P_{1}$ and $x_{0}=1-\mu$ for collisions with $P_{2}$. Let us remark that the determinant of the Levi-Civita matrix in (7) corresponds to the distance $R$ from the singularity, namely

$$
R \equiv u_{1}^{2}+u_{2}^{2}=\sqrt{\left(x-x_{0}\right)^{2}+y^{2}}
$$

so that $R=r_{1}$ if we regularize in $P_{1}$ and $R=r_{2}$ if we regularize in $P_{2}$. Then, we introduce a fictitious time $s$, which is related to the ordinary time $t$ through the expression

$$
d t=D d s
$$

where the quantity $D$ will be chosen later in order to regularize the equations of motion. We denote by a prime the derivative with respect to the regularized time. It is easy to show that:

$$
\begin{aligned}
\left(\begin{array}{l}
u_{1}^{\prime} \\
u_{2}^{\prime}
\end{array}\right) & =\frac{1}{2 R}\left(\begin{array}{rr}
u_{1} & u_{2} \\
-u_{2} & u_{1}
\end{array}\right)\left(\begin{array}{l}
x^{\prime} \\
y^{\prime}
\end{array}\right) \\
\left(\begin{array}{l}
u_{1}^{\prime \prime} \\
u_{2}^{\prime \prime}
\end{array}\right) & =-\frac{R^{\prime}}{2 R^{2}}\left(\begin{array}{rr}
u_{1} & u_{2} \\
-u_{2} & u_{1}
\end{array}\right)\left(\begin{array}{l}
x^{\prime} \\
y^{\prime}
\end{array}\right)+\frac{1}{2 R}\left(\begin{array}{rr}
u_{1}^{\prime} & u_{2}^{\prime} \\
-u_{2}^{\prime} & u_{1}^{\prime}
\end{array}\right)\left(\begin{array}{l}
x^{\prime} \\
y^{\prime}
\end{array}\right) \\
& +\frac{1}{2 R}\left(\begin{array}{rr}
u_{1} & u_{2} \\
-u_{2} & u_{1}
\end{array}\right)\left(\begin{array}{l}
x^{\prime \prime} \\
y^{\prime \prime}
\end{array}\right),
\end{aligned}
$$

where $R^{\prime}=2\left(u_{1} u_{1}^{\prime}+u_{2} u_{2}^{\prime}\right)$. Using the relation between the real and fictitious times one obtains:

$$
\left(\begin{array}{l}
x^{\prime \prime} \\
y^{\prime \prime}
\end{array}\right)=\dot{D}\left(\begin{array}{l}
x^{\prime} \\
y^{\prime}
\end{array}\right)+D^{2}\left(\begin{array}{l}
\ddot{x} \\
\ddot{y}
\end{array}\right) .
$$

Combining (8) and (9), the derivatives $u_{1}^{\prime \prime}$ and $u_{2}^{\prime \prime}$ are given by

$$
\begin{aligned}
& u_{1}^{\prime \prime}=\left(\frac{D^{\prime}}{D}-\frac{R^{\prime}}{R}\right) u_{1}^{\prime}+\frac{u_{1}^{\prime 2}+u_{2}^{\prime 2}}{R} u_{1}+\frac{D^{2}}{2 R}\left(u_{1} \ddot{x}+u_{2} \ddot{y}\right) \\
& u_{2}^{\prime \prime}=\left(\frac{D^{\prime}}{D}-\frac{R^{\prime}}{R}\right) u_{2}^{\prime}+\frac{u_{1}^{\prime 2}+u_{2}^{\prime 2}}{R} u_{2}+\frac{D^{2}}{2 R}\left(-u_{2} \ddot{x}+u_{1} \ddot{y}\right),
\end{aligned}
$$


where $\ddot{x}, \ddot{y}$ must be expressed in terms of the new variables. To this end, using the equations of motion (6) in cartesian coordinates, one obtains

$$
\begin{aligned}
\ddot{x} & =\frac{4}{D}\left(u_{1} u_{2}^{\prime}+u_{2} u_{1}^{\prime}\right)+u_{1}{ }^{2}-u_{2}{ }^{2}+x_{0} \\
& -(1-\mu) \frac{u_{1}{ }^{2}-u_{2}{ }^{2}+\mu+x_{0}}{r_{1}{ }^{3}}-\mu \frac{u_{1}{ }^{2}-u_{2}{ }^{2}+\mu-1+x_{0}}{r_{2}{ }^{3}} \\
& +F_{x}\left(u_{1}, u_{2}, u_{1}^{\prime}, u_{2}^{\prime}\right) \\
\ddot{y} & =-\frac{4}{D}\left(u_{1} u_{1}^{\prime}-u_{2} u_{2}^{\prime}\right)+2 u_{1} u_{2}-(1-\mu) \frac{2 u_{1} u_{2}}{r_{1}{ }^{3}}-\mu \frac{2 u_{1} u_{2}}{r_{2}{ }^{3}} \\
& +F_{y}\left(u_{1}, u_{2}, u_{1}^{\prime}, u_{2}^{\prime}\right),
\end{aligned}
$$

where the distances from the primaries are now given by

$$
\begin{aligned}
& r_{1}=\sqrt{\left(u_{1}^{2}-u_{2}^{2}+x_{0}+\mu\right)^{2}+\left(2 u_{1} u_{2}\right)^{2}} \\
& r_{2}=\sqrt{\left(u_{1}^{2}-u_{2}^{2}+x_{0}-1+\mu\right)^{2}+\left(2 u_{1} u_{2}\right)^{2}} .
\end{aligned}
$$

Next we choose the factor $D$ as $D=R$, which allows us to remove one of the singularities; denoting by $F_{u_{1}}, F_{u_{2}}$ the components of the dissipative force in the regularized variables, the equations of motion become :

- if we regularize in $P_{1}$ :

$$
\begin{aligned}
& u_{1}^{\prime \prime}=\left[\frac{1}{R}\left({u_{1}^{\prime}}^{2}+u_{2}^{\prime 2}-\frac{1-\mu}{2}\right)+\frac{R}{2}\left(R-\mu-\mu \frac{R-1}{r_{2}{ }^{2}}\right)\right] u_{1}+2 R u_{2}^{\prime}+F_{u_{1}} \\
& u_{2}^{\prime \prime}=\left[\frac{1}{R}\left(u_{1}^{\prime 2}+u_{2}^{\prime 2}-\frac{1-\mu}{2}\right)+\frac{R}{2}\left(R+\mu-\mu \frac{R+1}{r_{2}{ }^{3}}\right)\right] u_{2}-2 R u_{1}^{\prime}+F_{u_{2}} ;
\end{aligned}
$$

- if we regularize in $P_{2}$ :

$$
\begin{aligned}
& u_{1}^{\prime \prime}=\left[\frac{1}{R}\left(u_{1}^{\prime 2}+u_{2}^{\prime 2}-\frac{\mu}{2}\right)+\frac{R}{2}\left(R+1-\mu-(1-\mu) \frac{R+1}{r_{1}^{3}}\right)\right] u_{1}+2 R u_{2}^{\prime}+F_{u_{1}} \\
& u_{2}^{\prime \prime}=\left[\frac{1}{R}\left(u_{1}^{\prime 2}+u_{2}^{\prime 2}-\frac{\mu}{2}\right)+\frac{R}{2}\left(R-1+\mu-(1-\mu) \frac{R-1}{r_{1}^{3}}\right)\right] u_{2}-2 R u_{1}^{\prime}+F_{u_{2}} .
\end{aligned}
$$

The two cases can be written in compact form as

$$
\begin{aligned}
& u_{1}^{\prime \prime}=\left[\frac{1}{R}\left(u_{1}^{\prime 2}+u_{2}^{\prime 2}-\frac{1-\mu_{s}}{2}\right)+\frac{R}{2}\left(R+x_{0}-\mu_{s} \frac{R-x_{s}}{r_{s}^{3}}\right)\right] u_{1}+2 R u_{2}^{\prime}+F_{u_{1}} \\
& u_{2}^{\prime \prime}=\left[\frac{1}{R}\left(u_{1}^{\prime 2}+u_{2}^{\prime 2}-\frac{1-\mu_{s}}{2}\right)+\frac{R}{2}\left(R-x_{0}-\mu_{s} \frac{R+x_{s}}{r_{s}^{3}}\right)\right] u_{2}-2 R u_{1}^{\prime}+F_{u_{2}},
\end{aligned}
$$

where $r_{s}=r_{2}, \mu_{s}=\mu, x_{s}=1, x_{0}=-\mu$ if we regularize in $P_{1}$ and $r_{s}=r_{1}, \mu_{s}=1-\mu, x_{s}=$ $-1, x_{0}=1-\mu$ if we regularize in $P_{2}$. We conclude by providing the explicit expression of the dissipative terms as a function of the regularized variables, using the same regularization 
scheme; we also remark that the regularization itself is determined by the conservative part, where the strongest singularities occur. In particular, we obtain

$$
\begin{aligned}
& F_{u_{1}}=\frac{R}{2}\left(u_{1} F_{x}+u_{2} F_{y}\right) \\
& F_{u_{2}}=\frac{R}{2}\left(-u_{2} F_{x}+u_{1} F_{y}\right),
\end{aligned}
$$

where $\left(F_{x}, F_{y}\right)$ must be expressed as functions of the new variables. In the case of the linear drag we have

$$
\begin{aligned}
& F_{u_{1}}=-k \frac{R}{2}\left(2 u_{1}^{\prime}-u_{2}\left(R-x_{0}\right)\right) \\
& F_{u_{2}}=-k \frac{R}{2}\left(2 u_{2}^{\prime}+u_{1}\left(R+x_{0}\right)\right) .
\end{aligned}
$$

For the Stokes drag, if we denote by $\left(D_{x}, D_{y}\right)$ the components of the linear drag dissipation, the components $\left(S_{x}, S_{y}\right)$ of the Stokes drag are given by

$$
S_{x}=D_{x}-k \alpha \Omega y, \quad S_{y}=D_{y}+k \alpha \Omega x .
$$

It remains to transform the term $(-k \alpha \Omega y, k \alpha \Omega x)$ as

$$
\begin{aligned}
& F_{u_{1}}=-k \frac{R}{2}\left(2 u_{1}^{\prime}-u_{2}(1-\alpha \Omega)\left(R-x_{0}\right)\right) \\
& F_{u_{2}}=-k \frac{R}{2}\left(2 u_{2}^{\prime}+u_{1}(1-\alpha \Omega)\left(R+x_{0}\right)\right) .
\end{aligned}
$$

Concerning the Poynting-Robertson effect, let us remark that the expression of the force is the same as in the linear case except for the factor $1 / r_{1}^{2}$. Since PR is singular in $r_{1}$, we have two different cases:

- if we regularize in $P_{1}$ then:

$$
\begin{aligned}
& F_{u_{1}}=-\frac{k}{2 R}\left(2 u_{1}^{\prime}-u_{2}(R+\mu)\right) \\
& F_{u_{2}}=-\frac{k}{2 R}\left(2 u_{2}^{\prime}+u_{1}(R-\mu)\right) ;
\end{aligned}
$$

- if we regularize in $P_{2}$ then:

$$
\begin{aligned}
& F_{u_{1}}=-k \frac{R}{2 r_{1}^{2}}\left(2 u_{1}^{\prime}-u_{2}(R-1+\mu)\right) \\
& F_{u_{2}}=-k \frac{R}{2 r_{1}^{2}}\left(2 u_{2}^{\prime}+u_{1}(R+1-\mu)\right) .
\end{aligned}
$$




\section{References}

[1] C. Beaugé, S. Ferraz-Mello, Resonance Trapping in The Primordial Solar Nebula: The case of a Stokes Drag Dissipation, Icarus 103, 301-318 (1993)

[2] C. Beaugé, S. Ferraz-Mello, Capture in exterior mean-motion resonance due to PoyntingRobertson drag, Icarus 110, 239-260 (1994)

[3] C. Beaugé, S.J. Aarseth, S. Ferraz-Mello, Resonance capture and the formation of the outer planets, Month. Not. Royal Astr. Soc. 270, 21-34 (1994)

[4] J.A. Burns, P.L. Lamy, S. Soter, Radiation forces on small particles in the Solar System, Icarus 40, 1-48 (1979)

[5] A. Celletti, The Levi-Civita and $K S$ regularizing transformations, in "Singularities in Gravitational Systems", D. Benest, C. Froeschlé eds., Springer-Verlag, Berlin, Heidelberg, 1-24 (2002)

[6] C. Froeschlé, M. Guzzo, E. Lega, Graphical evolution of the Arnold's web: from order to chaos, Science 289, no. 5487, 2108-2110 (2000)

[7] C. Froeschlé, E. Lega, R. Gonczi, Fast Lyapunov indicators. Application to asteroidal motion, Cel. Mech. Dyn. Astr. 67, 41-62 (1997)

[8] R. Gonczi, Ch. Froeschlé, C. Froeschlé, Poynting-Robertson Drag and Orbital Resonance, Icarus 51, 633-654 (1982)

[9] J.D. Hadjidemetriou, George Voyatzis, On the dynamics of extrasolar planetary systems under dissipation: Migration of planets, Cel. Mech. Dyn. Astr. 107, 3-19 (2010)

[10] E. Lega, M. Guzzo, C. Froeschlé, Close encounters and resonances detection in threebody problems through Kustaanheimo Stiefel regularization, preprint (2010)

[11] J.C. Liou, H.A. Zook, A.A. Jackson, Radiation Pressure, Poynting-Robertson Drag and Solar Wind Drag in the Restricted Three Body Problem, Icarus 116, 186-201 (1995)

[12] C.D. Murray, S.F. Dermott, Solar System Dynamics, Cambridge University Press (1999)

[13] C.D. Murray, Dynamical effects of drag in the circular restricted three-body problem: I. Location and stability of the Lagrangian equilibrium points, Icarus 112, 465-484 (1994)

[14] P. Pástor, J. Klačka, L. Kómar, Motion of dust in mean motion resonances with planets, Cel. Mech. Dyn. Astr. 103, 343-364 (2009)

[15] B. Sicardy, C. Beaugé, S. Ferraz-Mello, D. Lazzaro, F. Roques, Capture of grains into resonances through Poynting-Robertson drag, Cel. Mech. Dyn. Astr. 57, 373-390 (1993) 
[16] M. Šilidichovskí, D. Nesvorný, Temporary capture of grains in exterior resonances with the Earth: Planar circular restricted three-body problem with Poynting-Robertson drag, Astron. \& Astroph. 289, 972-982 (1994)

[17] A. Sivasankaran, B.A. Steves, W.L. Sweatman, A global regularisation for integrating the Caledonian symmetric four-body problem, Cel. Mech. Dyn. Astr. 107, 157-168 (2010)

[18] E.L. Stiefel, G. Scheifele, Linear and Regular Celestial Mechanics, Springer-Verlag, Berlin, Heidelberg, New York (1971) 

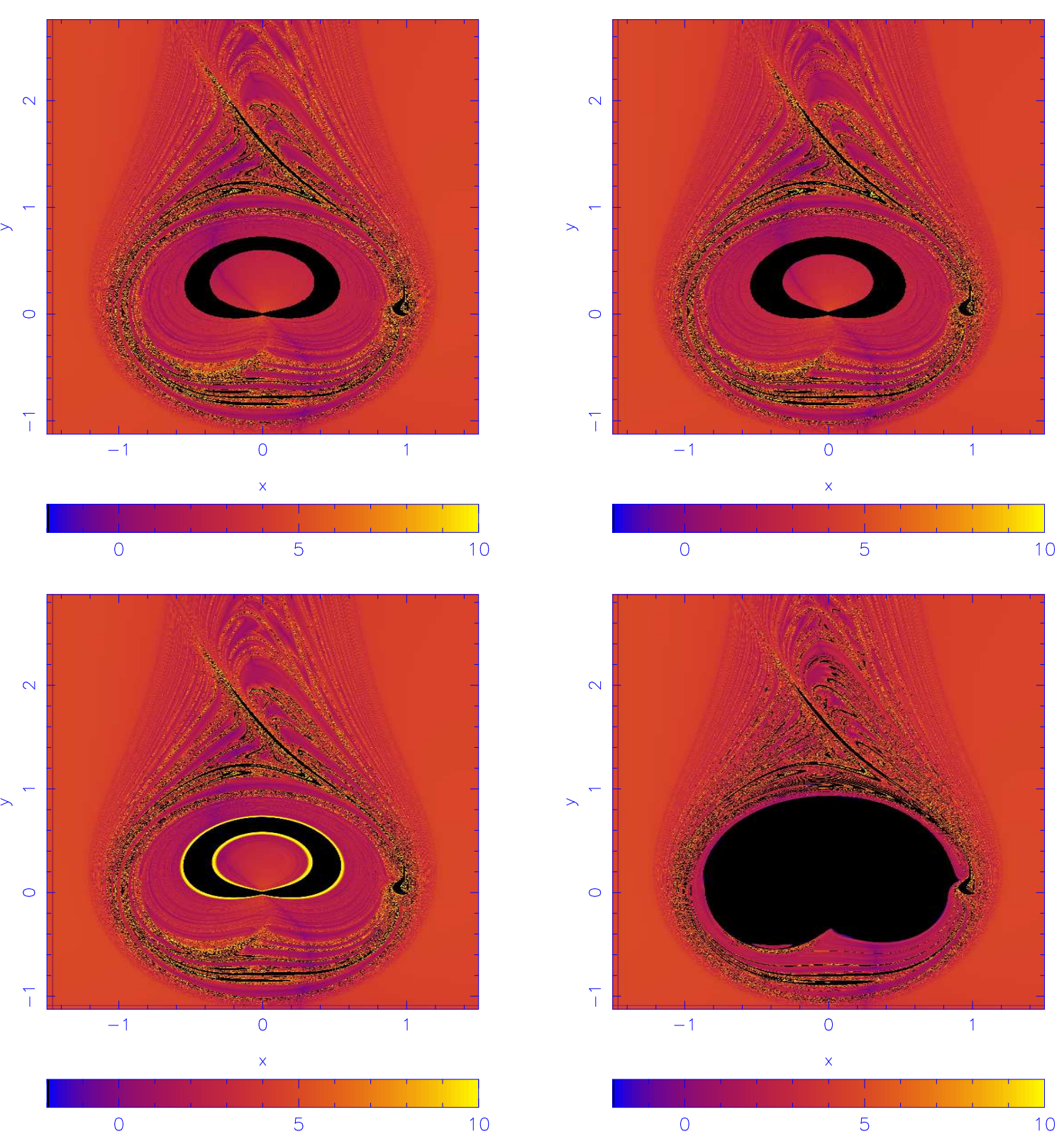

Figure 5: FLI map for the Stokes drag (top) for $\alpha=0.995$, and for the PR drag (bottom) with $T=100$. Left: $k=10^{-5}$, right: $k=10^{-3}$. 

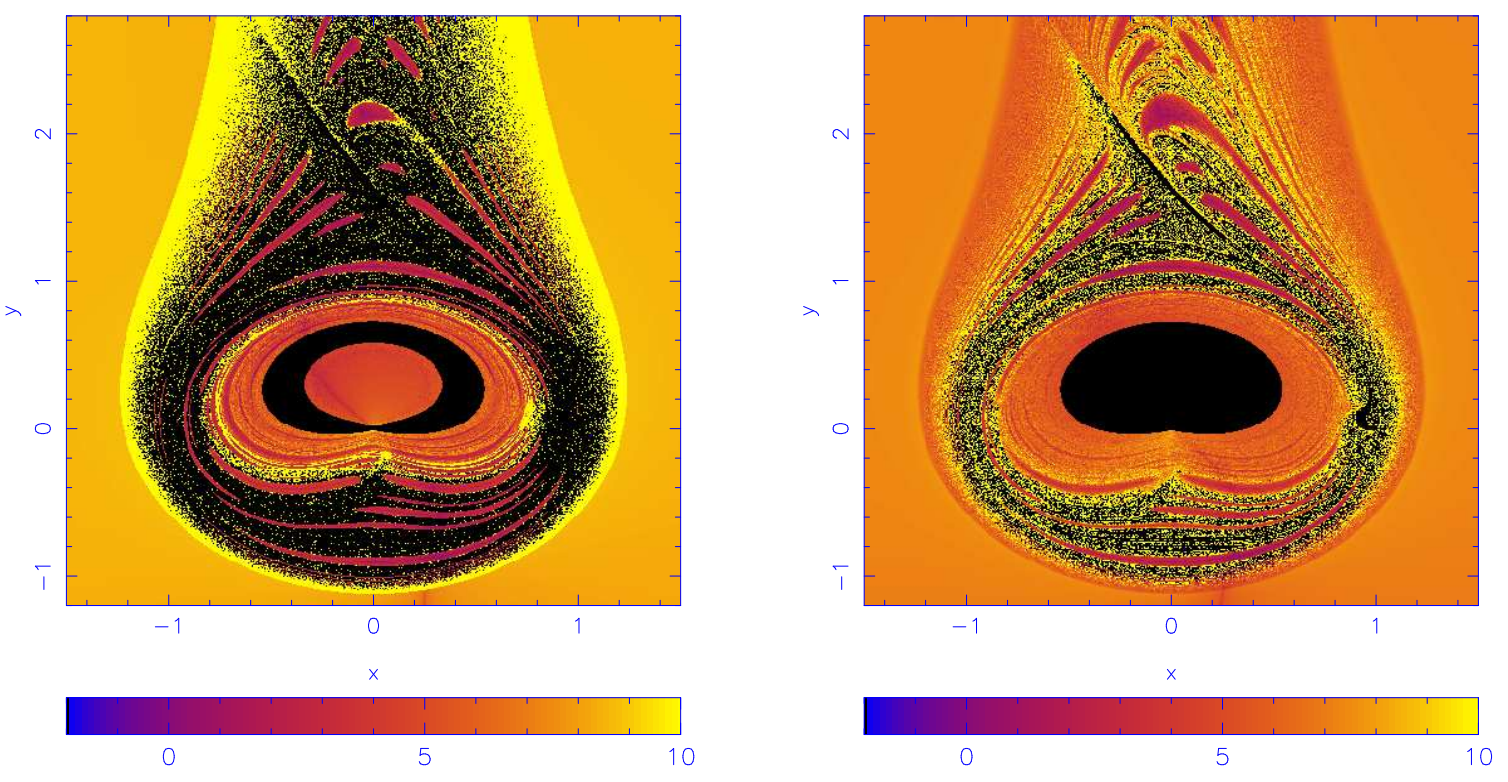

Figure 6: FLI map of the Stokes case with $\alpha=0.995$ and $T=5000$. Left: $k=10^{-5}$, right: $k=10^{-3}$. 

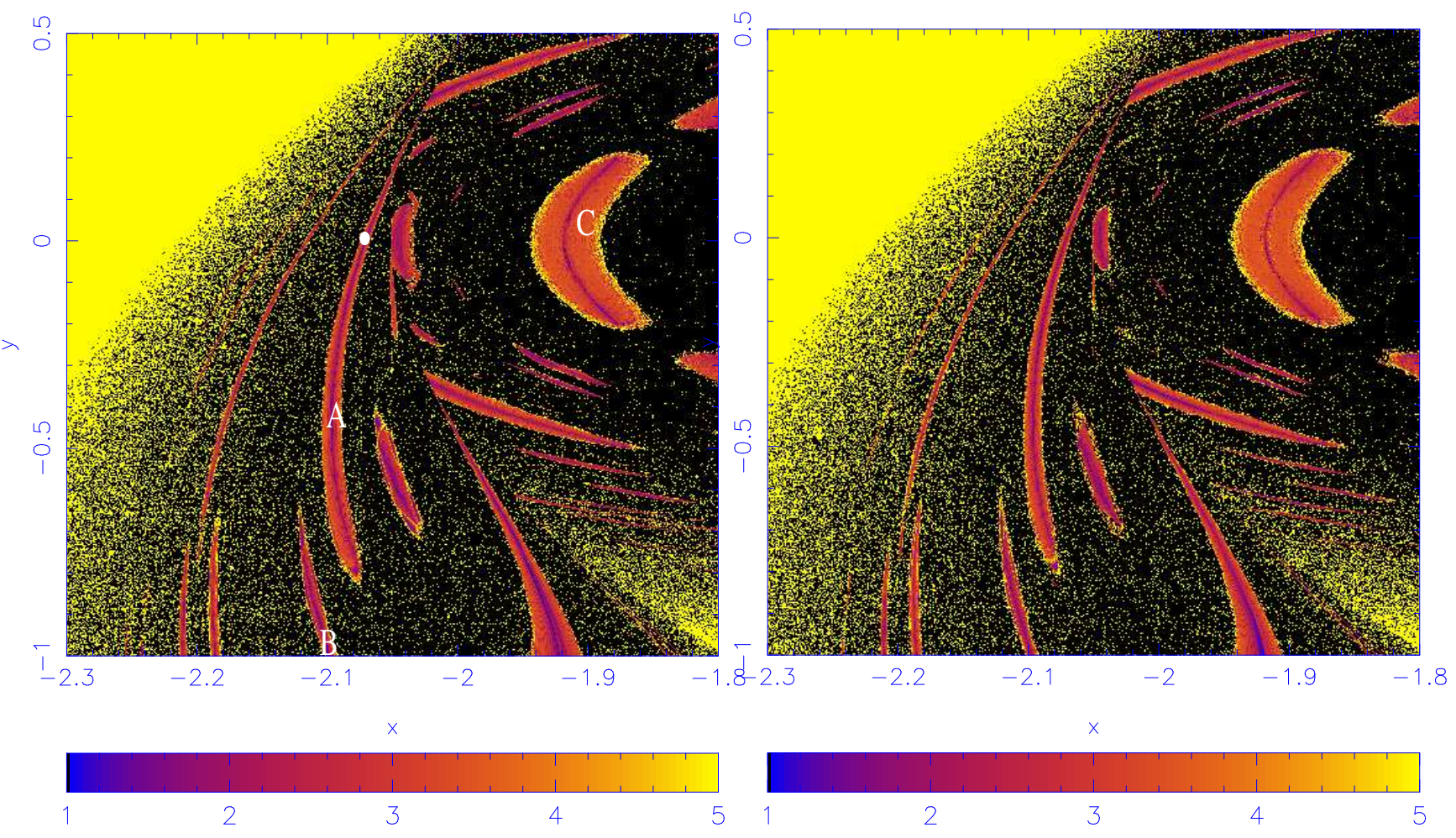

Figure 7: FLI map of the Stokes case for $T=5000$ in a neighborhood of the initial condition for the periodic orbit of period 3 (see Table 1 and Figure 3, left). Left: $\alpha=0.05$, right: $\alpha=0.995$. On the left panel the initial condition of the periodic orbit of period 3 is marked with a white circle. The letters A, B, C denote the initial conditions of Figure 8 and of Figure 9, top, middle and bottom panels, respectively. 

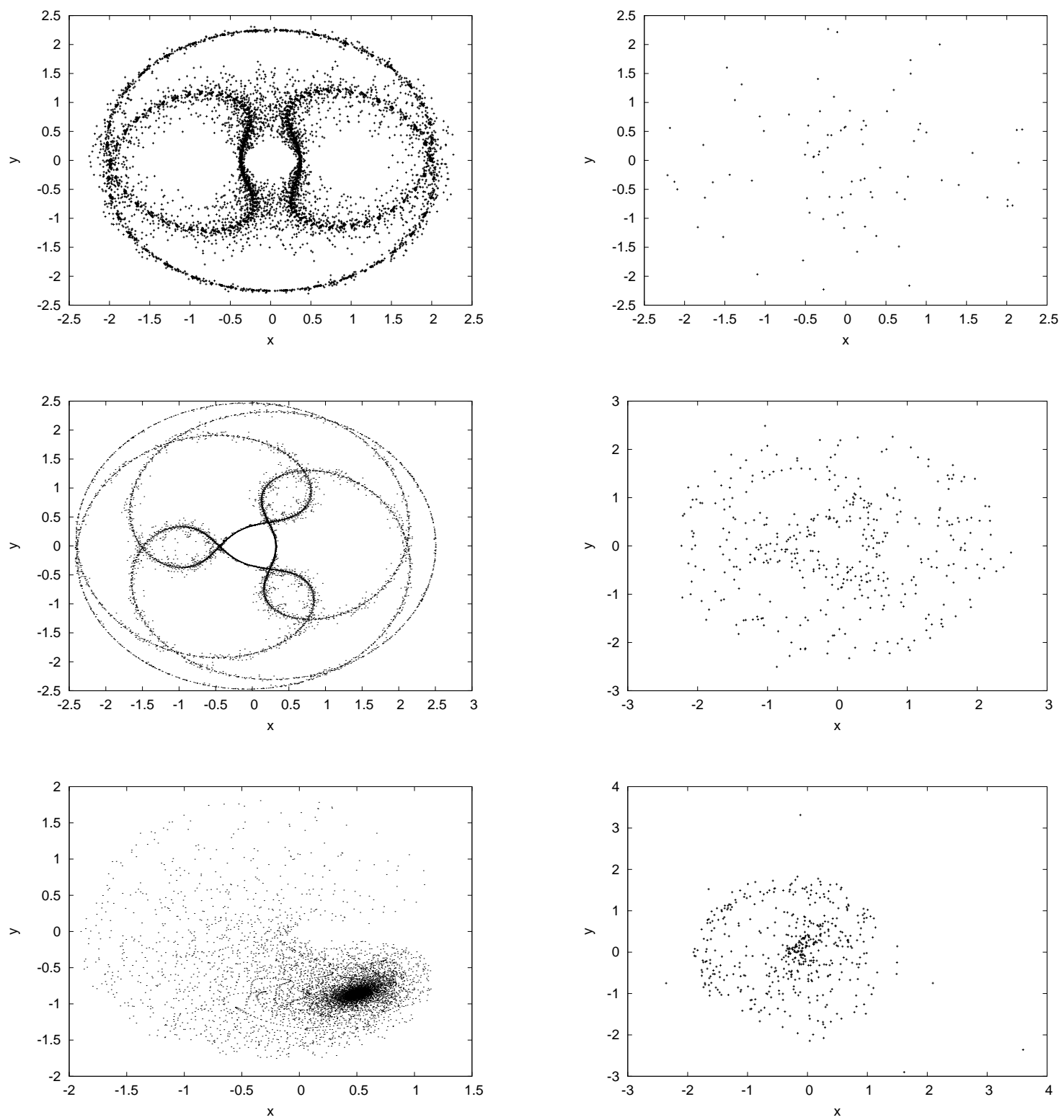

Figure 8: Orbits computed on a time $T=10^{6}$ and sampled every $\Delta t=100$ with initial conditions given in Table 2 (respectively, orbit A (top), B (middle), C (bottom)). Left column: $\alpha=0.05$, right column: $\alpha=0.995$. 

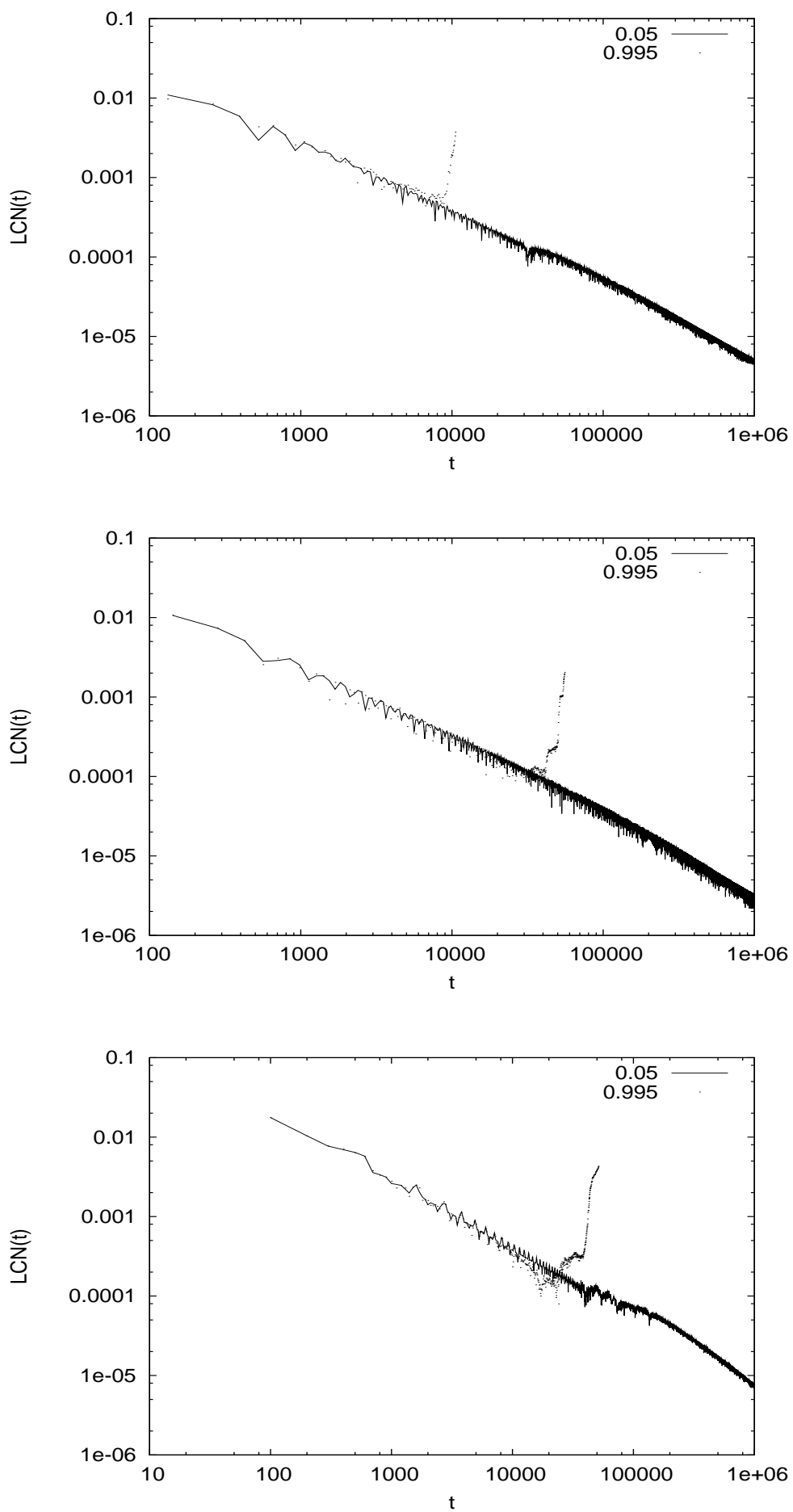

Figure 9: Computation of the largest Lyapunov indicator for the orbits with initial conditions given in Table 2 (respectively, orbit A (top), B (middle), C (bottom)) and plotted in Figure 8. The line corresponds to $\alpha=0.05$ and the dots correspond to $\alpha=0.995$. In the case $\alpha=0.995$ the computation is stopped, when the orbit escapes. 